\title{
Relationship between Breast, Formula and Combination Feeding on Childhood in Italy
}

\author{
A. Arrichiello ${ }^{1}$, F. Frezza ${ }^{2}$, G. Auriemma ${ }^{1}$, R. Palomba ${ }^{1}$, and F. Sarubbi ${ }^{1 *}$ \\ ${ }^{1}$ Institute for Animal Production System in Mediterranean Environment, National Research Council, Via Argine 1085, 80147, Naples, Italy \\ ${ }^{2}$ Pediatrician, dependent Local Health City of Naples, Italy
}

${ }^{*}$ Correspondence to: Fiorella Sarubbi, Institute for Animal Production System in Mediterranean Environment. National Research Council, Via Argine 1085, 80147, Naples, Italy; Fax: +39/0815965291; E-mail: fiorella.sarubbi@ispaam.cnr.it

Received: February 07, 2018; Accepted: February 21, 2018; Published: February 25, 2018;

\begin{abstract}
To investigate the relationship between breastfeeding, formula and combination feeding on weight childhood. The feeding group assignment was based as reported by medical records. Infant feeding was defined using three variables: breastfeeding duration, time until formula was introduced and infant weight. Number of Observations was equal to 294 ( $n=154$ girls and $n=140$ boys). The data were analyzed using the ANOVA procedure. Results were considered significant at $\mathrm{P}<.01$. The trend of the weight according to the feed type in the first 12 months of age between the data reported by the WHO and those collected. In addition, up to 4 months of life, to the growth curves appear similar to those reported by the WHO. In the first four months, about the girls and the boys, fed with milk formula grow equally. Differences are evident when subjects are fed at the breast. With breastfeeding, the differences between boys and girl are even more evident. The girls confirm the increased tendency to obesity than boy especially when breastfed. The results obtained different types of power supply could affect the tendency to overweight more in females than in males. The novelty of this study is focused on investigation about the relationship between breastfeeding, formula and combination feeding on weight in Italian childhood.
\end{abstract}

Keywords: breast; formula; childhood

\section{Introduction}

Breast milk provides an optimal source of nutrition for infants [1,2]. Many organizations, including the World Health Organization (WHO), recommend that healthy term infants be exclusively breastfed for the first 6 months of life [1,3,4]. This is, in part, because breast milk is easily digested and strengthens the infant's immune function $[3,5,6]$. Exclusive breastfeeding may also be modestly protective against excessive early infant gain and later obesity, whereas formula feeding may increase the risk of obesity in childhood [7]. Several biological mechanisms have been postulated to explain the potential differences between breastfed and formula fed infants - including postnatal effects of the intrauterine environment, the putative malleable metabolic environment in early infancy, and the permeability of the infant gut [8]. Another important benefit is the potential role of breastfeeding in preventing childhood obesity.

Melissa et al., [9] reported that breastfeeding, in the absence of formula feeding, appears to have a protective effect on childhood obesity. While combination feeding confers less benefit than exclusive breastfeeding, it is more desirable than formula feeding alone.

The findings underscore a need to support strategies and social policies that promote exclusive and longer breastfeeding duration as part of comprehensive strategies to prevent excess body weight and to reduce the burden of chronic diseases in the long term.

Potential biological explanations of the breastfeed obesity association have centered on differences in the nutritional composition of human milk versus formula, focusing in particular on the comparisons of human milk and formula in terms of protein and fatty acid composition [8]. For example, differences in the ratio of omega 3 and omega 6 fatty acids between human milk and formula have been associated with adipose tissue growth in infants [10] and insulin response in animal models [11]. A causal role for milk composition in promoting or protecting against obesity beyond infancy, however, has yet to be established [12].

Both maternal and infant behaviors differ based on whether an infant was fed from the breast [direct breastfeeding] or bottle-fed human milk and/or formula. It was suggested that direct breastfeeding supports the development of appetite regulation given its inherent dependence on infant-centered feeding behaviors by the mother [13]. This process relies heavily on the infant's response to satiation rather than on visual cues, as a breastfeeding mother does not know how much milk is being offered, how much the infant is taking in and also knows little about the rate with which their infant is drinking. Thus, direct breastfeeding requires the mother to focus on infant cues to gauge the child's interest in feeding (e.g. increased alertness to caregiver, moving head towards caregiver) and satiety (e.g. reduced sucking, drowsiness, relaxed state, arms close to the body). In contrast, bottle-feeding, involving either human milk or formula, provides explicit visual information about infant intake to the caregiver based on the amount of milk or formula remaining in the bottle.

Controlling feeding practices in infancy have an impact on children's weight at 2 years. The use of restrictive child feeding 
practices during infancy predicts lower child weight at age 2 years, which may reinforce mothers' use of this strategy in the longer term despite its potential association with disinhibition and greater child weight in later childhood [14].

Fisher et al., [15] found that prolonged breast-feeding throughout the first year of life was associated with higher toddler energy intake at age 18 months, and that this relationship was mediated by breastfeeding mothers reporting that they were less over-controlling when feeding their infants (i.e. pressuring to eat and restricting food less). Moreover, this relationship was found to be independent of the potentially confounding variables of maternal education, child gender and weight. Over controlling feeding techniques such as pressuring the child to eat or restricting child intake of foods are associated with greater feeding problems; pressure to eat is associated with reduced food preference, whilst restriction (generally of fat laden foods) is conversely linked with greater intake of the restricted foods [15]. Presumably, the impact of breast-feeding, mediated by maternal control, upon toddler energy intake, is accounted for by fewer aversive feeding interactions and less child refusal of food, but no data are available to explore this potential explanation.

This study aim to investigate the relationship between breastfeeding, formula and combination feeding on weight childhood.

\section{Methods}

This study employed a retrospective cohort design. Infant feeding group assignment was based as reported by medical records. Child growth data were assessed via medical records.

Infant feeding was defined using three variables:

1) Breastfeeding duration;

2) Time until formula was introduced.

3) Infant weight

Have been taken consider two modes of infant feeding:

- Breast

- Formula

Infant feeding was then categorized by examining the response to both variables. Exclusive breastfeeding, defined by the WHO as no other food or drink except breast milk for the first 6 months of life, could not be determined in this study because of the lack of information around introduction to solids. Many of the children were both breastfed and formula fed, thus, a combination variablerepresenting breastfeeding together with formula feeding-was derived. Only formula fed was considered for mothers who breastfed for a 1 week or never. Standing height was measured, without shoes, to the nearest $0.1 \mathrm{~cm}$ and body weight to the nearest 0.1 kilogram on calibrated digital scales.

Descriptive statistics were used to characterize the population by weight status. In univariate regression we evaluated the association between breastfeeding duration and weight status as well as the association between the combination feeding variable and weight status. Odds ratios were calculated separately for normal weight versus overweight/obese, normal weight versus obese and normal weight versus overweight. ANOVA was carried out to evaluate significant differences between the two feedings (breast $v s$ formula). The Pearson's correlation was used to evaluate the relationship among parameters. The data were analyzed using the General Linear Models procedure of Ref. [16].

\section{Results and Discussion}

Table 1 presents a description of the sample.

Table 1: Number of observations (n) of type of feeding, all genres, ranges of observation age.

\begin{tabular}{|c|c|c|}
\hline Feeding & Number of observation (n) & $\%$ \\
\hline Breast & 109 & 37.1 \\
\hline Formula & 185 & 62.9 \\
\hline Genres & 294 & \\
\hline Girl & 154 & 52.4 \\
\hline Boy & 140 & 47.6 \\
\hline Range of observation age & 294 & \\
\hline $1-3$ months & 88 & 29.9 \\
\hline $4-6$ months & 52 & 17.7 \\
\hline $10-12$ months & 36 & 12.2 \\
\hline Over 12 months & 118 & 40.1 \\
\hline
\end{tabular}

An examination of table 1 clearly shows that $32 \%$ of the samples analyzed was fed with formula, $18 \%$ was fed at the breast, while about $3 \%$ had a mixed feeding. Children fed with formula or with mixed feeding were weaning with other milk at the age of four months, while those fed breast were weaning at the age of six months.

In Table 2 we report the correlation matrix among the type of feed and weight of infants.

Table 2: Correlation matrix among the type of feeding and weight of infants.

\begin{tabular}{|l|l|c|c|}
\hline & & Feeding & Weight \\
\hline Feeding & Pearson Corr. & 1 & $-0.222(* *)$ \\
\hline & Sig. &. & .007 \\
\hline Weight & Pearson Corr. & $-0.222(* *)$ & \\
\hline & Sig. & .007 & \\
\hline
\end{tabular}

** Correlation is significant at the 0.01 level.

Table 2 shows that were the negative correlation between types of feeding and weigh of infants.

Jing Yan et al., [17] reported a protective effect of breastfeeding for childhood obesity, and prolonged breastfeeding is directly related to a decreasing risk of obesity. In particularly, children being breastfed for $\geq 7$ are significantly less likely to be obese in later childhood.

Figure 1 shows the trend of the weight according to the feed type in the first 23 months of age between the data reported by the WHO and those collected in the present study (Figure 1).

Figure 1 shows that up to 4 months of life, leaving aside the type of power supply, to the growth curves appear similar to those reported by the WHO. Then the difference becomes increasingly apparent with increasing age. This difference can be justified by the fact that in Italy, probably, it are anticipated, often, the age of weaning age resulting in greater weight gain. 
In Figure 2 we reported the increase in weight in function of age by the different types of feeding.

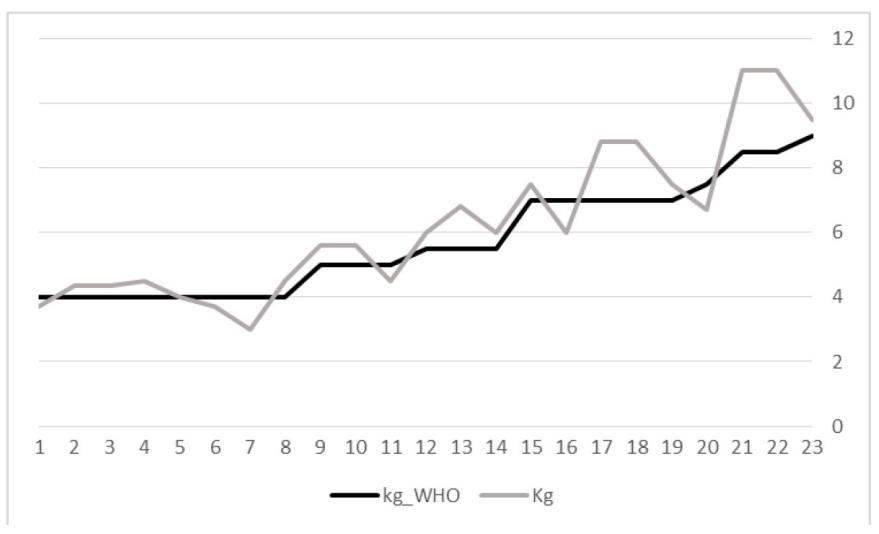

Figure 1: Trend of the weight according to the breast feed type in the first 23 months of age between the data reported by $\mathrm{WHO}$ and those collected in the present study.

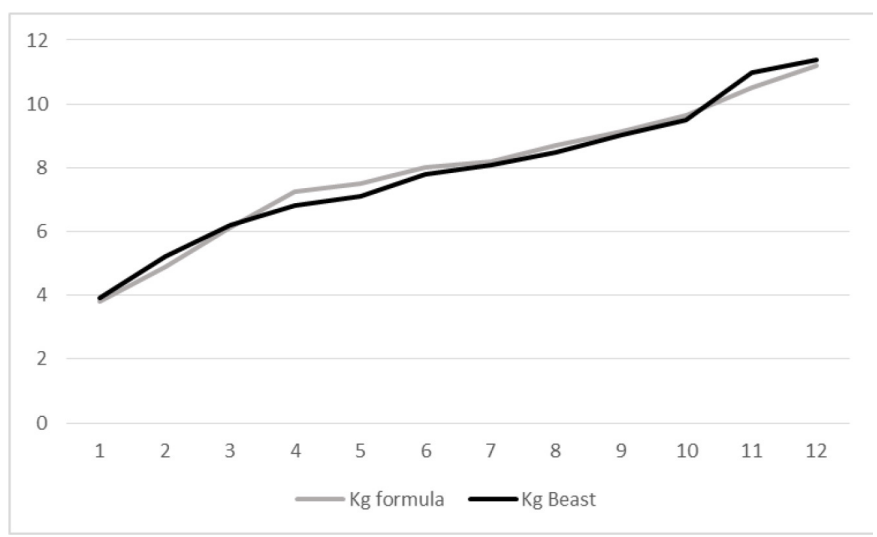

Figure 2: Mean weight curves according to feeding status in function of age (until 12 months).

There were no significant differences between different feeding. In the first 4 months, the increase in weight appears to be faster in those who are breastfed with formula than those fed to the breast. This trend disappears in the following months.

In Figure 3 reported the trend of the weight according to the feed with formula in the first 12 months of age according to the gender.

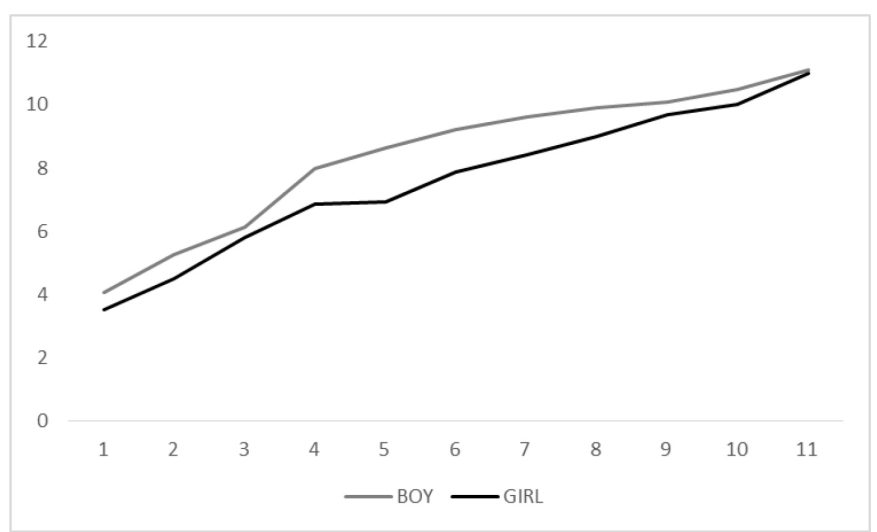

Figure 3: Trend of the weight according to the feed with formula in the first 12 months of age according to the gender.
Figure 3 shows that the trend of grow was equally in the boy and girl. The boy shows that an increased weight in every control. This difference can be justified by the different metabolism begins to diversify between boy and girl.

Figure 4 shows the trend of the weight according to the feed with formula in the first 12 months of age according to the gender.

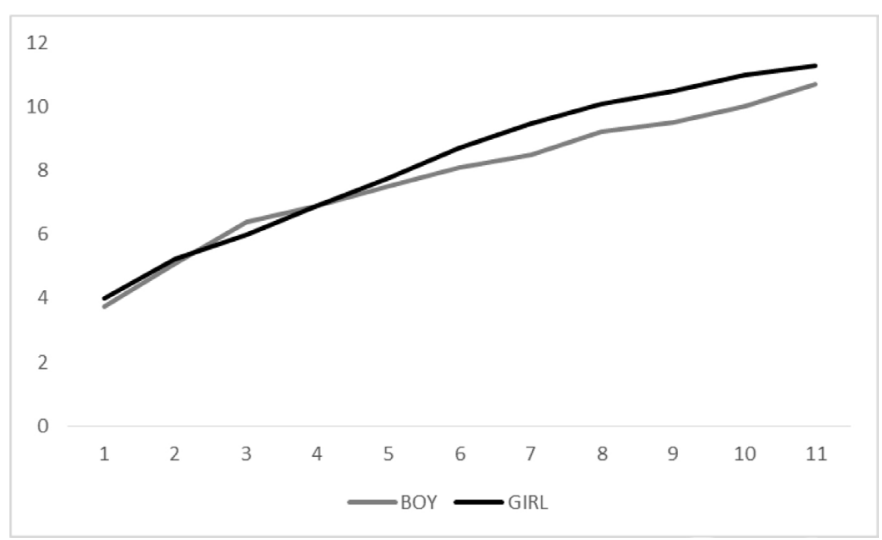

Figure 4: Trend of the weight according to the feed with breast in the first 12 months of age according to the gender.

The figures shows, also, that while in boy the increased weight trend is linear, both with breast than with the milk formula, in girl, feeding with the formula, appears more swinging in the first months of life. This trend does not take statistical significance, so it can be considered random.

From the figures we can see that those who were breastfed show an increase in more weight than those fed with formula. Also the trend is not very linear. The increase in weight is linear in individuals breastfed than those fed with formula, almost overlapping in the final part of the graph. If we compare Figure 3 and 4 , in the first 4 months of age, we can see that the girl confirm the increased tendency to overweight than boy especially when formula.

We can conclude that the results obtained different types of power supply could affect the tendency to overweight more in girl than in boy. It is also clear that such tendency toward also depends on other cofactors which may act by increasing and / or decreasing this risk.

\section{Implications}

There are several studies aimed to investigate the relationship between breastfeeding duration, combination feeding on overweight and obesity in children. Other papers reported that excessive protein intake in the first two years of infants' life - potentially through baby formula - could be contributing to childhood obesity. The novelty of this study is focused on investigation about the relationship between breastfeeding, formula and combination feeding on weight in Italian childhood. Differently to other works found in literature, this study has pointed its attention on the different trend of the increase in children's weight according to the sex.

\section{Acknowledgements}

We are grateful to Mr. Giuseppe Grazioli for his technical support. 


\section{References}

1. World Health Organization (2011) Media Centre Statement 15 January 2011. Exclusive breastfeeding for six months best for babies everywhere http: //www. who.int/mediacentre/news/statements/2011/breastfeeding_20110115/en/

2. Twells L, Newhook LA, Ludlow V (2012) Can breastfeeding reduce the risk of childhood obesity? In S. A. Yuca (Ed.), Childhood Obesity pp. 53-78.

3. Health Canada, Canadian Paediatric Society, Dietitians of Canada, Breastfeeding Committee for Canada (2012) Nutrition for healthy term infants: Recommendations from birth to six months. Food and Nutrition. Retrieved from Health Canada website: http: //www.hc-sc.gc.ca/fn-an/nutrition/infant-nourisson/recom/indexeng,php\#a5.

4. Kramer MS, Kakuma R (2012) Optimal duration of exclusive breastfeeding. Cochrane Database System Review (8): CD003517.

5. Duijts L, Jaddoe VWV, Hofman A, Moll HA (2010) Prolonged and exclusive breastfeeding reduces the risk of infectious diseases in infancy. Pediatrics 126(1): $18-25$.

6. Heinig MJ (2001) Host defense benefits of breastfeeding for the infant: Effect of breastfeeding duration and exclusivity. Pediatric Clinics of North America 48(1): 105-123.

7. Li R, Fein S, Grummer-Strawn LM (2010) Do infants fed from bottles lack selfregulation of milk intake compared with directly breastfed infants? Pediatrics 125(6): 1386-1393.

8. Young BE, Johnson SL, Krebs NF (2012) Biological determinants linking infant weight gain and child obesity: Current knowledge and future directions. Advantes Nutrition 3(5): 675-686.

9. Rossiter MD, Colapinto CK, Khan MKA, McIsaac JLD, Williams PL, et al. (2015) Breast, Formula and Combination Feeding in Relation Childhood Obesity in Nova Scotia, Canada Matern Child Health J 19: 2048-2056.

10. Horta BL, Bahl R, Martines JC, Victoria CG (2007) Evidence on the long-term effect of breastfeeding: Systematic reviews and meta-analyses. Retrieved from World Health Organization website: http://www.who.int/child_adolescent_health/ documents/9241595230/en/index.html

11. Owen CG, Martin RM, Whincup PM, Davey-Smith G, Gillman MW, Cook DG (2005) The effect of breastfeeding on mean body mass index throughout life: Quantitative review of published and unpublished odservational evidence. The American Journal of Clinical Nutrition 82(6): 1298-1307.

12. Stolzer JM (2011) Breastfeeding and obesity: A meta-analysis. Open Journal of Preventive Medicine 1: 88-93.

13. Monasta L, Batty GD, Cattaneo A, Lutje V, Ronfani L, et al. (2010) Early-life determinants of overweight and obesity: a review of systematic reviews. Obes Rev 11: 695-708. [Crossref]

14. Farrow CV, Blissett J (2008) Controlling feeding practices: cause or consequence of early child weight? Pediatrics 121: e164-169. [Crossref]

15. Fisher JO, Birch LL, Smiciklas-Wright H, Picciano MF (2000) Breast-feeding through the first year predicts maternal control in feeding and subsequent toddler energy intakes. Journal of the American Dietetic Association 100: 641-646.

16. SPSS 12.0 Package software (2003)

17. Jing Yan, Lin Liu, Yun Zhu, Guowei Huang, Peizhong Peter Wang (2014) The association between breastfeeding and childhood obesity: a meta-analysis. $B M C$ Public Health 14: 1267-1277.

Citation:

A. Arrichiello, F. Frezza, G. Auriemma, R. Palomba, and F. Sarubbi (2018) Relationship between Breast, Formula and Combination Feeding on Childhood in Italy. Endocrinol Diabetes Metab J Volume 2(2): 1-4 